García Perales, R., Jiménez Fernández, C., y Palomares Ruiz, A. (2020). Seguimiento de un grupo de alumnos y alumnas con alta capacidad matemática. Revista de Investigación Educativa, 38(2), 415-434.

DOI: http://dx.doi.org/10.6018/rie.366541

\title{
Seguimiento de un grupo de alumnos y alumnas con alta capacidad matemática
}

\section{Monitoring of a group of students with high mathematical ability}

\author{
Ramón García Perales*, Carmen Jiménez Fernández** y Ascensión Palomares Ruiz* \\ *Departamento de Pedagogía. Facultad de Educación de Albacete. Universidad de Castilla-La Mancha (UCLM). España \\ **Departamento de MIDE I. Facultad de Educación. Universidad Nacional de Educación a Distancia (UNED). España
}

\begin{abstract}
Resumen
El presente estudio tiene como finalidad realizar un seguimiento de veintidós estudiantes diagnosticados como de alta capacidad matemática en el curso 2011/2012 cuando estudiaban $5^{\text {o }}$ de Educación Primaria. Forman el grupo situado en el nivel superior de la Batería de Evaluación de la Competencia Matemática (BECOMA, García-Perales, 2014), instrumento utilizado para decidir el grado de capacidad matemática de los estudiantes evaluados. En el curso 2017/2018 se han recogido nuevos datos sobre los veintidós estudiantes a fin de analizar sus trayectorias personales y académicas y comprobar el grado de estabilidad del diagnóstico realizado. Se trata de un estudio ex post facto, descriptivo y de corte cuantitativo. Los resultados indican que el grupo mantiene la alta capacidad matemática seis años después lo que viene a confirmar la validez diagnóstica de la BECOMA si bien la tendencia observada en los datos originales es un ligero descenso en los resultados en 2017/2018. No hay diferencias estadísticamente significativas entre alumnas y alumnos. La trayectoria escolar es importante también en estos escolares y su atención educativa debería ser más visible.

Palabras clave: diagnóstico educativo; rendimiento académico; alta capacidad; competencia matemática; indicadores de alta competencia matemática.
\end{abstract}

Correspondencia: Ramón García Perales, Ramon.GarciaPerales@uclm.es, Plaza de la Universidad, 3, 02071 Albacete (España). 


\begin{abstract}
The purpose of this study is to monitor twenty-two students diagnosed as having high mathematical ability in the 2011/2012 academic year, when they were studying 5th year of Primary Education. They form the group positioned at the highest level of the Mathematical Competence Assessment Battery (BECOMA, García-Perales, 2014), an instrument used to decide the degree of mathematical ability of the students evaluated. In the 2017/2018 academic year, new data about the twenty-two students were collected in order to analyze their personal and academic trajectories and verify the degree of stability of the diagnosis made. It is an ex post facto, descriptive and quantitative study. The results indicate that the group maintains the high mathematical capacity six years later, which confirms the diagnostic validity of the BECOMA although the observed empirical trend is a slight decline in the results in 2017/2018. There are no statistically significant differences between male and female students. The school trajectory is also important in these students, and their educational attention should be more visible.

Keywords: educational diagnosis; academic performance; high capacity; mathematical competence; indicators of high mathematical competence.
\end{abstract}

\title{
Introducción
}

Los procesos educativos incluyen retos sociales que generan un debate alrededor de la definición de cuáles deberían de ser los aprendizajes fundamentales a adquirir por el alumnado a lo largo de la escolarización obligatoria, haciendo indispensable la integración del trabajo por competencias en las programaciones didácticas (Méndez, Méndez, \& Fernández-Río, 2015) y considerando la formación docente un pilar básico, decisivo incluso, de la calidad del proceso (Escudero, González, \& Rodríguez, 2018).

La Organización para la Cooperación y el Desarrollo Económico (OCDE) comenzó en 1997 el proyecto PISA (Program for International Student Assessment). En él, la competencia matemática se constituye como área de evaluación, siendo valorada de manera prioritaria en las ediciones de 2003 y 2012. En la edición de 2012 se define como (Instituto Nacional de Evaluación Educativa -INEE-, 2013, pp. 12-13):

Capacidad del individuo para formular, emplear e interpretar las Matemáticas en distintos contextos. Incluye el razonamiento matemático y la utilización de conceptos, procedimientos, datos y herramientas Matemáticas para describir, explicar y predecir fenómenos. Ayuda a los individuos a reconocer el papel que las Matemáticas desempeñan en el mundo y a emitir los juicios y las decisiones bien fundadas que los ciudadanos constructivos, comprometidos y reflexivos necesitan.

En los centros educativos existen escolares con rendimiento excelente en la competencia matemática. PISA 2012, última edición que la evalúa de forma prioritaria, y PISA 2015, última edición de PISA, conceptualizan como sigue el rendimiento superior o nivel 6 de la competencia matemática en la escala PISA (INEE, 2013, p. 42): 
Los alumnos y alumnas saben formar conceptos, generalizar y utilizar información basada en investigaciones y modelos de situaciones de problemas complejos. Pueden relacionar diferentes fuentes de información y representaciones y traducirlas entre ellas de manera flexible. Pueden aplicar su entendimiento y comprensión, así como su dominio de las operaciones y relaciones Matemáticas simbólicas y formales y desarrollar nuevos enfoques y estrategias para abordar situaciones nuevas. Pueden formular y comunicar con exactitud sus acciones y reflexiones relativas a sus descubrimientos, interpretaciones, argumentos y su adecuación a las situaciones originales.

Por su parte, la Batería de Evaluación de la Competencia Matemática (BECOMA, GarcíaPerales, 2014) define como sigue su nivel de rendimiento superior o nivel 7:

Posible escolar con alta capacidad y talento matemático. Utiliza la información de forma sistemática y fluida, aplicándola con eficacia. Planifica, ejecuta y resuelve de manera creativa tareas con distintos niveles de complejidad. Asume riesgos y toma decisiones. Puede llegar a resolver el $100 \%$ de las tareas asignadas. Reconoce y contextualiza los problemas planteados, identifica sus componentes y sus interrelaciones, establece estrategias para su resolución y sabe justificar lo realizado. Aplica los conocimientos aprendidos a una situación planteada y reflexiona sobre sus relaciones internas. Gran dominio de las operaciones básicas y de las estrategias de cálculo mental.

Como todos los estudiantes, los que tienen potencialidades superiores deben participar de una educación inclusiva de calidad. Algunas investigaciones afirman que en las escuelas españolas apenas existen escolares con rendimiento bueno o strong perfomers, nivel 5 de PISA, y muy bueno o top performers, nivel 6 de PISA (García-Perales \& Jiménez, 2016; INEE, 2013; OCDE, 2009, 2013, 2016), extremo del que sería principal responsable el sistema en sí que no logra desarrollar la capacidad y el talento (matemático) allí donde existe.

Todo proceso educativo deberá estar guiado por los ejes calidad-equidad-educación (Torres \& Castillo, 2016), donde además de las aptitudes cobren importancia las actitudes. En el caso de la competencia matemática existen investigaciones que señalan que el interés y la motivación de los alumnos y alumnas por las Matemáticas es bajo y que disfrutan poco con su aprendizaje (Cerda et al., 2016; De La Rosa, 2007; Cleary \& Chen, 2009; OCDE, 2013), existiendo un componente afectivo en su adquisición que el profesorado debería considerar (Cerda, Pérez, Romera, \& Casas, 2017; Mato, Espiñeira, \& Chao, 2014). Es decir, el profesorado deberá de hacer uso de métodos de enseñanza que contemplen las actitudes del alumnado (García-Perales, 2017; Gasco, 2016; Gutiérrez-Gutiérrez, Gómez, \& Rico, 2016; Muñoz \& Mato, 2008; Nortes \& Nortes, 2016), y las expectativas hacia los estudiantes que en ocasiones existen y que pueden incidir en su rendimiento en la área de Matemáticas (Tourón, Lizasoaín, Castro, \& Navarro, 2012). La trascendencia de esta área es obvia y su gene- 
ralización a la vida cotidiana justifica el aprendizaje de sus elementos, algoritmos y lógica. Centros y profesorado deben crear oportunidades reales para su aprendizaje y desarrollar indicadores de proceso de aula capaces de mostrar qué se hace en los centros y aulas con resultados buenos y menos buenos, y qué oportunidades de aprendizaje ofrecen en cada caso.

A partir de la toma en consideración de las aptitudes y actitudes para la enseñanza y el aprendizaje de la matemática, inicia el seguimiento de un grupo de escolares que en el curso 2011/2012 fueron diagnosticados como estudiantes con alta capacidad matemática. En 2017/2018 han vuelto a ser evaluados con vistas a analizar su trayectoria personal y académica y observar, al mismo tiempo, el grado de estabilidad del diagnóstico realizado seis años antes.

\section{Método}

Este artículo expone parte de los datos obtenidos en el seguimiento de 22 estudiantes situados en el nivel 7, el nivel superior de la BECOMA, que les fue administrada en 2011/2012 cuando cursaban quinto curso de Educación Primaria. Está formada por 34 reactivos que puntúan con 0,1 ó 2 puntos, y 8 pruebas denominadas: Interpretación matemática (5 ítems), Cálculo mental (6), Propiedades geométricas (2), Series lógicas numéricas (6), Descubriendo algoritmos (2), Unidades convencionales (6), Series lógicas de figuras (3) e Invención de problemas (4). Una publicación anterior (García-Perales \& Jiménez, 2016) profundiza en la citada batería y en las variables medidas entonces a dicho grupo. En el curso 2017/2018 han vuelto a ser evaluados como pasamos a exponer.

Se trata de una investigación ex post facto, descriptiva y de corte cuantitativo que recoge medidas repetidas de una muestra de 22 estudiantes de la provincia de Albacete, que en el curso académico 2017/2018 estudiaban 4º de Educación Secundaria Obligatoria (ESO) o $1^{\underline{0}}$ de Bachillerato.

\section{Objetivos}

El objetivo principal es iniciar el seguimiento de este grupo de escolares de rendimiento matemático superior, comprobar la consistencia actual del diagnóstico respecto al realizado en 2011/2012 y analizar sus trayectorias académicas y personales y sus posibles modulaciones. Se traduce en los siguientes objetivos:

a) Identificar la consistencia de los resultados en las medidas repetidas en los cursos 2011/2012 y 2017/2018.

b) Estudiar la congruencia de los resultados de 2017/2018 en la prueba de capacidad matemática BADyG-S en comparación con los obtenidos en 2011/2012 en el BADyG-E3 y en la BECOMA.

c) Observar a lo largo del estudio si existen diferencias significativas según la variable sexo. 


\section{Población y Muestra}

La muestra la componen los 22 escolares diagnosticados con alta capacidad matemática en 2011/2012. En el momento del diagnóstico estaban escolarizados en $5^{\mathrm{o}}$ de Educación Primaria y fueron seleccionados de una muestra estratificada de 712 escolares representativa de la población de ese nivel para la provincia de Albacete, equivalía al 17,94\% de la población escolar. Son el 3,1\% de los 712 estudiantes que respondieron a la BECOMA y sus puntuaciones en ella oscilaron entre 59 y 68, siendo 68 la puntuación techo. Las características distintivas de estos escolares se resumen en la tabla 1.

De forma resumida, estas características fueron: sexo, 14 alumnos $(63.64 \%)$ y 8 (36.36\%) alumnas; entorno, 18 escolares (81.81\%) en centros urbanos y $4(18.19 \%)$ en rurales; titularidad del centro, 20 escolares (91.91\%) en centros públicos y 2 (9.09\%) en privados-concertados; no existencia de repetidores de curso; rendimiento académico en el área de Matemáticas, 18 escolares tenían sobresaliente, 3 notable y 1 bien; interés y motivación hacia el área de Matemáticas según el punto de vista del docente, con mucho interés 19 escolares, 2 bastante y 1 regular; interés y motivación hacia el área de Matemáticas según el punto de vista del propio escolar, 16 escolares tenían mucho interés y 6 bastante; elevada aptitud matemática a juicio del tutor, 8 escolares (36\%) no tenían elevada aptitud matemática y 14 sí; BADyG-E3, puntuación centil media de 92 y un CI promedio de 141; alta capacidad detectada, de los 22 escolares, 2 contaban con un diagnóstico de altas capacidades intelectuales realizado por los Servicios de Orientación de sus centros educativos.

\section{Variables e Instrumentos}

Se exponen las variables medidas indicando a su vez los instrumentos utilizados. El tutor ha sido el encargado de recopilar la información de cada escolar. Las medidas repetidas en los dos momentos han sido las siguientes:

- Sexo: Hombre o Mujer.

- Entorno del centro: Urbano o Rural.

- Titularidad del centro: Público o Privado-concertado.

- Repetición de curso: Sí o No.

- Rendimiento académico. Insuficiente: 1, Suficiente: 2, Bien: 3, Notable: 4, y Sobresaliente: 5. Se anota el rendimiento académico en el área de Matemáticas en ambos períodos, siempre a fecha de junio.

- Interés del escolar hacia el área de Matemáticas según el docente. Nada: 1, Poco: 2, Regular: 3, Bastante: 4, y Mucho: 5.

- Interés del escolar hacia el área de Matemáticas según el propio estudiante. Nada: 1, Poco: 2, Regular: 3, Bastante: 4, y Mucho: 5.

- Existencia o no de elevada aptitud matemática en el escolar según el docente. Sí o No.

- Alta capacidad detectada. Existencia de diagnóstico por parte de un Servicio de Orientación público: Sí o No. 


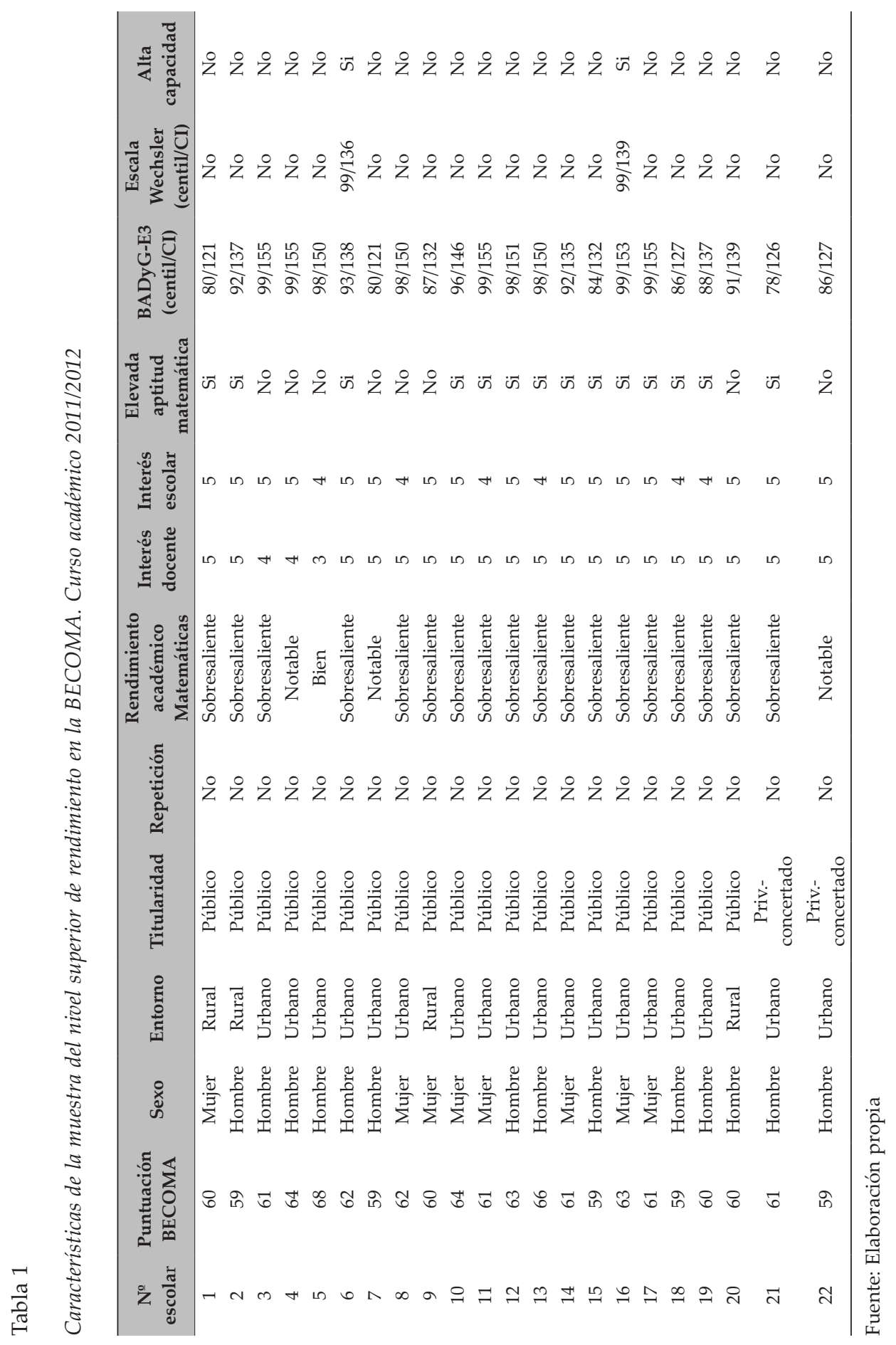


- BADyG-E3 (2011/2012) y BADyG-S (2017/2018). Capacidad matemática medida por las subpruebas Series numéricas (Rn) y Problemas numéricos (Sn) de ambos instrumentos. Ofrecen puntuaciones centiles y Cociente Intelectual (CI). Se han usado ambas puntuaciones en ambas subpruebas, y se ha obtenido una tercera variable mediante el promedio de los resultados obtenidos en ambas, base de la exposición de los resultados. Son pruebas psicopedagógicas que ofrecen perfiles cognitivos sobre la inteligencia general y los factores numérico, verbal, espacial y lógico, memoria, atención y rapidez y eficacia en la ejecución.

- WISC-IV. Escala de Inteligencia Wechsler (2005) que ofrece puntuación centil y CI. La había aplicado el Servicio de Orientación a 5 de los 22 estudiantes y decidimos incorporarla. Consta de 15 pruebas que evalúan capacidades intelectuales agrupadas en cuatro dimensiones: comprensión verbal, razonamiento perceptivo, memoria de trabajo y velocidad de procesamiento.

\section{Procedimiento de recogida y análisis de datos}

Usaremos estadísticos descriptivos, índices de correlación y contraste de diferencias para muestras relacionadas, comparando los resultados alcanzados en 2017/2018 con los obtenidos en 2011/2012 (meses de marzo a junio de cada curso), para ver si las diferencias habidas en las medidas tienen o no significación estadística. Diferencias no significativas en capacidad matemática pueden tomarse como estabilidad del diagnóstico realizado en 2011/2012. También puede valorarse el grado de estabilidad de las opiniones y juicios de estudiantes y profesorado sobre la capacidad matemática al comparar ambos momentos. Las posibles diferencias por género se analizarán a lo largo del estudio.

\section{Resultados}

Los resultados se exponen siguiendo los objetivos formulados:

a) Consistencia de los resultados en las medidas repetidas en 2011/2012 y 2017/2018.

b) Congruencia de los resultados de 2017/2018 en el BADyG-S en comparación con los obtenidos en 2011/2012 en el BADyG-E3 y en la BECOMA.

c) Comportamiento de la variable sexo.

La tabla 2 recoge los datos de las medidas recogidas en 2017/2018: 


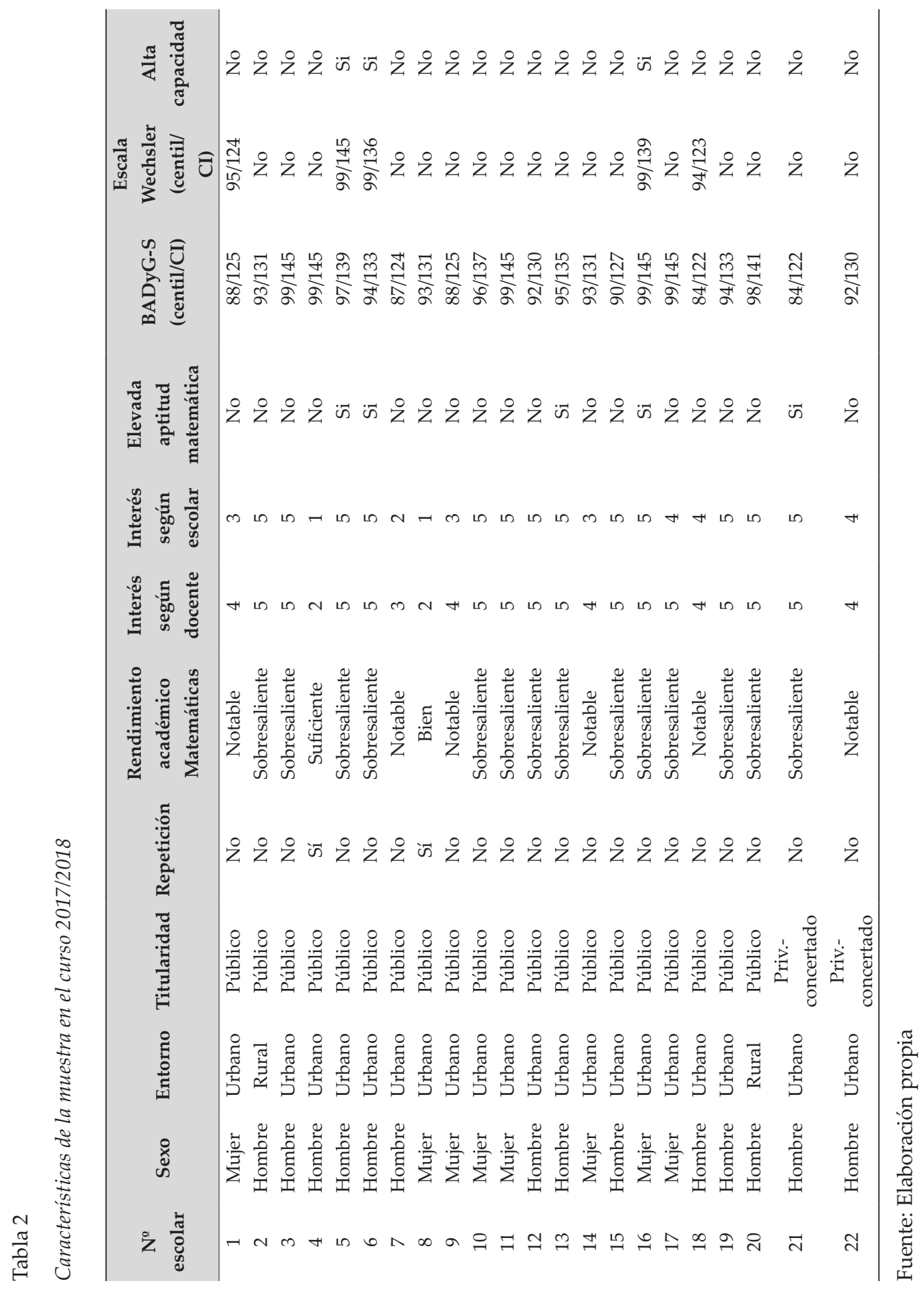




\section{a) Consistencia de los resultados en las medidas repetidas en los cursos 20 I I/20 I 2 y $2017 / 2018$}

La tabla 3 recoge los estadísticos descriptivos de las variables medidas en los dos momentos.

Tabla 3

Estadísticos descriptivos para las medidas en los cursos 2011/2012 y 2017/2018

\begin{tabular}{|c|c|c|c|c|c|c|c|c|c|c|c|c|}
\hline \multirow{2}{*}{ Variables } & \multicolumn{6}{|c|}{ 2011/2012 } & \multicolumn{6}{|c|}{$2017 / 2018$} \\
\hline & Mín & Máx & $M$ & DT & Asim & Curt & Mín & Máx & $M$ & DT & Asim & Curt \\
\hline $\begin{array}{l}\text { Rendimiento } \\
\text { académico }\end{array}$ & 3 & 5 & 4.77 & .53 & -2.39 & 5.46 & 2 & 5 & 4.50 & .80 & -1.83 & 3.48 \\
\hline Matemáticas & & & & & & & & & & & & \\
\hline $\begin{array}{l}\text { Interés } \\
\text { docente }\end{array}$ & 3 & 5 & 4.82 & .50 & -2.91 & 8.43 & 2 & 5 & 4.41 & .96 & -1.67 & 2.00 \\
\hline $\begin{array}{l}\text { Interés } \\
\text { escolar }\end{array}$ & 4 & 5 & 4.73 & .46 & -1.10 & -.89 & 1 & 5 & 4.09 & 1.34 & -1.35 & .72 \\
\hline $\begin{array}{l}\text { Elevada } \\
\text { aptitud } \\
\text { matemática }\end{array}$ & 1 & 2 & 1.36 & .49 & .61 & -1.80 & 1 & 2 & 1.77 & .43 & -1.40 & -.06 \\
\hline Entorno & 1 & 2 & 1.18 & .39 & 1.77 & 1.25 & 1 & 2 & 1.09 & .29 & 3.06 & 8.08 \\
\hline Repetición & 2 & 2 & 2 & --- & --- & --- & 1 & 2 & 1.91 & .29 & -3.06 & 8.08 \\
\hline $\begin{array}{l}\text { Alta } \\
\text { capacidad } \\
\text { detectada }\end{array}$ & 1 & 2 & 1.91 & .29 & -3.06 & 8.09 & 1 & 2 & 1.86 & .35 & -2.28 & 3.50 \\
\hline Sexo & 1 & 2 & 1.36 & .49 & .61 & -1.80 & 1 & 2 & 1.36 & .49 & .61 & -1.80 \\
\hline Titularidad & 1 & 2 & 1.09 & .29 & 3.06 & 8.09 & 1 & 2 & 1.09 & .29 & 3.06 & 8.08 \\
\hline
\end{tabular}

Fuente: Elaboración propia

En esta tabla se muestra que de 2011/2012 a 2017/2018 decae levemente el rendimiento académico medio en Matemáticas (media pasa de 4.77 a 4.50), así como el grado de interés por ellas según la valoración del docente (media cambia de 4.82 a 4.41) y de los propios estudiantes (media baja de 4.73 a 4.09) y el número de estudiantes señalados por sus profesores como de alta aptitud matemática (14 considerados afirmativamente en el primer periodo y 5 en el segundo). En 2017/2018 aparecen dos estudiantes repetidores. En todos estos casos en 2017/2018 ha aumentado la dispersión o desviación típica de los datos. Por su parte, en 2011/2012 habían diagnosticado a dos estudiantes como de alta capacidad; en 2017/2018 tres. No ha habido cambios en las frecuencias de sexo y titularidad de centro.

Las diferencias observadas entre ambos momentos del estudio se han sometido a contraste mediante la prueba t para las variables ordinales o continuas, y a la prueba ji-cuadrado para las variables dicotómicas. 
Tabla 4

Prueba t para muestras relacionadas

\begin{tabular}{lccccc}
\hline Variables & gl & Valor & Sig. & Correlación & Sig. $^{2}$ \\
\hline $\begin{array}{l}\text { Rendimiento académico } \\
\text { Matemáticas }\end{array}$ & 21 & 1.55 & .14 & .28 & .20 \\
$\begin{array}{l}\text { Interés docente } \\
\text { Interés escolar }\end{array}$ & 21 & 1.82 & .08 & .06 & .78 \\
& 21 & 2.08 & .06 & -.03 & .88 \\
\hline
\end{tabular}

Fuente: Elaboración propia

Según la tabla 4, las diferencias observadas no son estadísticamente significativas en ninguna de las tres características medidas si bien como hemos apuntado, la tendencia observada en los datos originales es hacia un ligero descenso en 2017/18 en las variables medidas en los dos momentos. En el caso del rendimiento académico un escolar ha pasado de bien a sobresaliente, otro ha pasado de notable a suficiente y un tercero baja de sobresaliente a bien. En el caso de las alumnas en cuatro de ellas, el $50 \%$, ha descendido el rendimiento matemático. También se aprecia un ligero declive en interés y motivación hacia el área de Matemáticas según el punto de vista del propio escolar y en interés y motivación hacia el área de Matemáticas y en elevada aptitud matemática según el punto de vista del docente. Se intuyen dificultades en los docentes para la identificación de estos estudiantes. Por otra parte, algunos estudios afirman que baja el rendimiento académico del alumnado en el tránsito de las etapas educativas (Mato, Espiñeira, \& Chao, 2014), siendo más preocupante en el caso de la población femenina por su mayor número y por la aparición de ansiedad y falta de confianza (INEE, 2013), extremos hacia los que apuntan nuestros datos, tal y como se ha observado, por ejemplo, para la variable rendimiento académico.

Respecto al interés y motivación hacia el área de Matemáticas según el punto de vista del docente, llama la atención el escolar número 5, véase Tablas 1 y 2, que en 2011/2012 fue calificado con un interés y motivación regular y seis años después es definido con mucho interés, y en 2017/2018 ha sido diagnosticado como de altas capacidades. En 2011/2012, los estudiantes fueron calificados 19 con mucho interés, 2 con bastante y 1 regular. Seis años después han bajado y hay 14 escolares en la opción mucho, 5 en bastante, 1 en regular y 2 en poco, los dos últimos son los estudiantes repetidores que han pasado de bastante interés y motivación a tener poco.

Finalmente, en interés y motivación hacia el área de Matemáticas según el punto de vista del propio escolar, destacar los escolares 4 y 8 , que en un primer momento mostraban un interés y motivación de mucho y bastante, y que actualmente es de nada. Algo similar ocurre con el escolar número 7, cuya autopercepción pasa de mucho a poco. En 2011/2012, la distribución era de 16 dentro de mucho y 6 para bastante; en 2017/2018, 13 se incluyen en la opción de mucho, 3 en bastante, 3 en regular, 1 en poco y 2 en nada. Resultados coincidentes con otros estudios que con- 
cluyen que las actitudes de los estudiantes hacia esta área son importantes, inciden en el rendimiento y el autoconcepto y deben de tenerse presente en la planificación y desarrollo de las programaciones didácticas del área de Matemáticas (Cueli, García, \& González-Castro, 2013; INEE, 2013; Mato, Espiñeira, \& Chao, 2014; Tourón, Lizasoaín, Castro, \& Navarro, 2012).

Para las variables dicotómicas aparece lo siguiente:

Tabla 5

Prueba de ji-cuadrado para variables dicotómicas

\begin{tabular}{lcc}
\hline Variables & Valor & Sig. \\
\hline Elevada aptitud matemática & .75 & .38 \\
Entorno & 9.90 & .00 \\
Repetición & --- & --- \\
Alta capacidad detectada & .75 & .39 \\
Sexo & --- & --- \\
Titularidad & --- & --- \\
\hline
\end{tabular}

Fuente: Elaboración propia

La variable entorno, tal y como se refleja en la tabla 6, es la única que arroja diferencias estadísticamente significativas. Ello es debido a que en 2011/2012 había 4 escolares en centros rurales y 18 en urbanos; en 2017/2018 había 2 estudiantes en centros rurales y 20 en urbanos.

En elevada aptitud matemática a juicio del docente, en 2011/2012, 14 estudiantes fueron considerados de elevada aptitud matemática (2 de ellos habían sido diagnosticados con altas capacidades intelectuales por el Servicio de Orientación escolar) y 8 no; en 2017/2018 solo 5 estudiantes fueron valorados positivamente (tres de ellos habían sido diagnosticados con altas capacidades por los Servicios de Orientación) y 17 no. La precisión de los juicios evaluativos del profesor en este campo es una cuestión lábil y compleja y, en todo caso, es visible la necesidad de mejorar su formación (Escudero, González, \& Rodríguez, 2013, 2018; Zeichner, Payne, \& Brayko, 2015) tanto en el campo de la matemática (Gutiérrez-Gutiérrez, Gómez, \& Rico, 2016; INEE, 2013; Nortes \& Nortes, 2013, 2016; Rico, Gómez, \& Cañadas, 2014) como en el de los más capaces (García-Perales, 2017).

En cuanto a la repetición de curso, en 2011/2012 ningún escolar había repetido. En 2017/2018 aparecen dos repetidores, los escolares número 4 en 2º de la ESO (CI 155 en el BADYG E-3) y la número 8 en 1ํ de la ESO (CI 150 en el BADYG-E3).

Respecto a la alta capacidad detectada, en el 2011/2012 la muestra tenía dos escolares que habían sido diagnosticados con altas capacidades intelectuales por los Servicios de Orientación; en 2017/2018 había aumentado a tres. Los estudiantes diagnosticados por la BECOMA con alta capacidad matemática no han sido considerados como tales por los Servicios de Orientación escolar. En los seis años que median entre ambas evaluaciones, solo el escolar número 5 que en 2011/2012 obtuvo 
rendimiento académico de bien y la mayor puntuación del curso en la BECOMA, ha sido diagnosticado como tal, lo que unido a los dos identificados con anterioridad a esa fecha, sitúa la prevalencia del diagnóstico oficial en tres casos o el 13.64\% de los 22 escolares ubicados en el nivel 7 o nivel superior de competencia matemática. Ninguno de los 22 estudiantes participa en programas de enriquecimiento, flexibilización curricular o similar. Esta escasez de estudiantes diagnosticados con alta capacidad es un aspecto sensible a revisar en la praxis educativa (Almeida \& Oliveira, 2010; Gagné, 2015; Muñoz \& Espiñeira, 2010; Sastre, 2014). Por otra parte, la "congruencia entre el sistema de identificación y la intervención posterior es tan importante, que podría ser considerada la 'regla de oro' de la educación del alumnado con alta capacidad intelectual" (Renzulli \& Gaesser, 2015, p. 104) ¿No tenemos alumnos capaces o se los traga el sistema?

\section{b) Congruencia de los resultados de 2017/2018 en el BADyG-S en comparación con los obtenidos en 20I I/20I 2 en el BADyG-E3 y en la BECOMA}

En el BADyG-S aplicado en 2017/2018, la muestra obtiene resultados similares a los alcanzados en el BADyG-E3 en 2011/2012, tal y como puede observarse en la Figura 1:

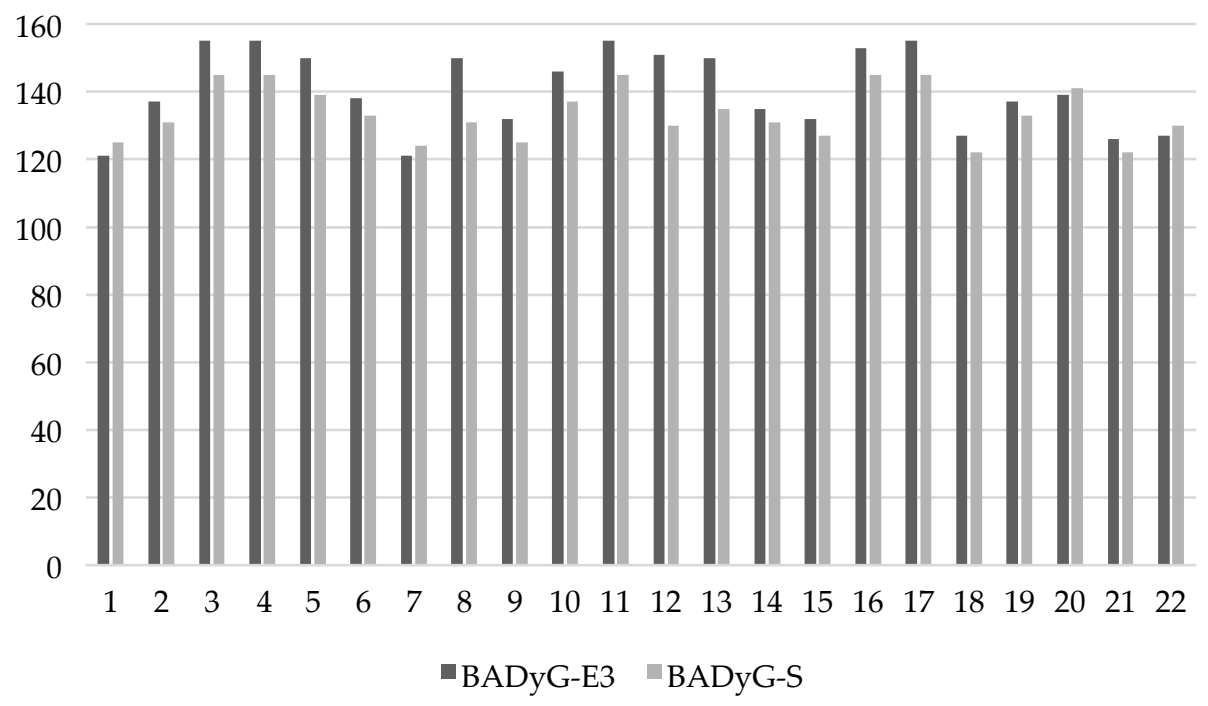

Figura 1. Cociente intelectual del alumnado en el BADyG-E3 y en el BADyG-S

Fuente: Elaboración propia 
Tabla 6

Estadísticos para ambas pruebas psicopedagógicas

\begin{tabular}{lcccccc}
\hline Prueba & Mín & Máx & M & DT & Asim & Curt \\
\hline BADyG-E3 & 121 & 155 & 140.55 & 11.85 & -.21 & -1.39 \\
BADyG-S & 122 & 145 & 133.68 & 8.01 & .20 & -1.23 \\
\hline
\end{tabular}

Fuente: Elaboración propia

En la tabla 6 se observan las diferencias y similitudes en los resultados para ambos instrumentos y se aprecia la tendencia empírica a un ligero descenso de los resultados medios en el curso 2017/2018 respecto a 2011/2012, incluso cuando se mide la capacidad con la ๑misma® prueba (de ahí la alta correlación) solo que adaptada para un grupo de mayor edad. Sin embargo, las diferencias habidas entre ambos resultados no son significativas estadísticamente como muestra la prueba $\mathrm{t}, \mathrm{t}(1,21)=4.91(\mathrm{p}<.001)$, correlación .85 ( $\mathrm{p}<.001)$.

La Figura 2 muestra los resultados obtenidos en la BECOMA en 2011/12 y los obtenidos en el BADyG-S seis años después. Se hace uso de puntuaciones centiles. Recordemos que ambas pruebas miden la competencia matemática y que el BADyGE3 fue uno de los criterios usados para validar la BECOMA.

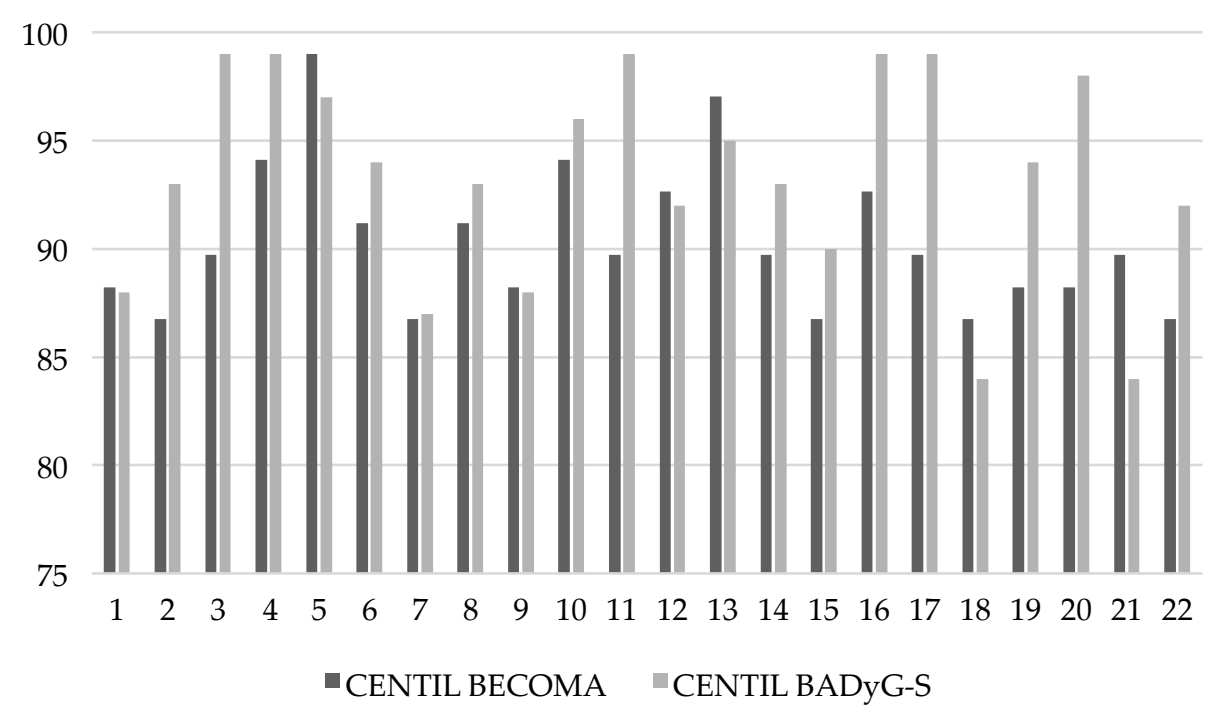

Figura 2. Resultados en la BECOMA y en el BADyG-S

Fuente: Elaboración propia 
Tabla 7

Estadísticos para la BECOMA y el BADyG-S

\begin{tabular}{lcccccc}
\hline Prueba & Mín & Máx & M & DT & Asim & Curt \\
\hline BECOMA & 59 & 68 & 61.45 & 2.40 & 1.24 & 1.37 \\
BADyG-S & 122 & 145 & 133.68 & 8.01 & .20 & -1.23 \\
\hline
\end{tabular}

Fuente: Elaboración propia

En la Figura 2 se observan puntuaciones centiles elevadas y similares para ambos instrumentos. De acuerdo a la tabla 7, podemos observar las puntuaciones numéricas para la BECOMA, oscilan entre 59 y 68 y con una media de $61.45(\mathrm{DT}=2.40)$ y de CI para el BADyG-S, intervalo de 122 a 145 y con una media de $133.68(\mathrm{DT}=8.01$ ). Tras contrastar los resultados del BADyG-S con los obtenidos en su momento en la BECOMA, la prueba $\mathrm{t}, \mathrm{t}(1,21)=-46.60(\mathrm{p}<.001)$, correlación .44 $(\mathrm{p}<.05)$ indica que las diferencias son estadísticamente significativas, lo que puede interpretarse como un indicador positivo de la validez de la BECOMA. Señalar que 16 escolares obtienen un CI promedio de 130 o superior en el BADyG-S, y que los 3 estudiantes diagnosticados con altas capacidades por los Servicios de Orientación escolar obtienen puntuaciones de CI de 139, 133 y 145 en dicho instrumento.

En cuanto a la Escala WISC-IV recordemos que había sido aplicada a cinco escolares a iniciativa de los Servicios de Orientación. De ellos, dos habían sido diagnosticados con altas capacidades intelectuales con anterioridad a la aplicación de la BECOMA en 2011/2012, y uno ha sido diagnosticado con posterioridad y antes de que recogiéramos los datos en 2017/2018 (Véase tablas 1 y 2). Tras la aplicación de la BECOMA, se informó a los centros educativos de los resultados obtenidos por los 22 estudiantes con rendimiento superior. Sin embargo, los centros han realizado evaluación psicopedagógica aplicando la Escala Wechsler WISC-IV en algún momento de su proceso educativo solo a tres de ellos, confirmando el diagnóstico de altas capacidades en el caso del escolar número 5 (CI Escala Wechsler 145, CI BADYG-E3 150, CI BADyG-S 139). Sorprende que no realizaran evaluación psicopedagógica a 17 de los 22 estudiantes de rendimiento superior en la BECOMA que tenían, además, resultados elevados en diferentes indicadores de actitud y aptitud, como el BADyGE3 y, actualmente, en el BADyG-S. En el caso de estas últimas pruebas, de los 17 escolares que obtuvieron en 2011/2012 un CI de 130 o superior en el BADYG-E3, solo 3 aparecen diagnosticados con altas capacidades intelectuales actualmente, con CI en el BADyG-S de 139, 133 y 145.

\section{c) Comportamiento de la variable sexo a lo largo del estudio}

En ambos momentos de la investigación se mantienen fijas las frecuencias muestrales o 14 alumnos (63.64\%) y 8 alumnas (36.36\%). La tabla 8 refleja el comportamiento de la variable sexo con el resto de variables para el segundo momento del estudio: 
Tabla 8

Prueba t para muestras independientes según el sexo, curso 2017/2018

\begin{tabular}{lcccc}
\hline \multirow{2}{*}{ Variables } & \multicolumn{2}{c}{ Estadístico de Levene } & \multicolumn{2}{c}{ Prueba $\mathbf{t}$} \\
\cline { 2 - 5 } & $\mathbf{F}$ & Sig. & $\mathbf{t}$ & Sig. \\
\hline Rendimiento académico Matemáticas & .01 & .95 & .54 & .59 \\
Interés docente & .02 & .90 & .58 & .57 \\
Interés escolar & .33 & .57 & 1.25 & .23 \\
Elevada aptitud matemática & 3.59 & .08 & -.84 & .41 \\
Repetición & .64 & .43 & .40 & .69 \\
Alta capacidad detectada & .06 & .82 & -.11 & .91 \\
BADyG-S & .61 & .44 & -.80 & .43 \\
\hline
\end{tabular}

Fuente: Elaboración propia

No aparece significación estadística en ninguna de las variables analizadas. Si comparamos estos resultados con de 2011/2012, tenemos lo siguiente (García-Perales, 2014): Rendimiento académico Matemáticas $\mathrm{t}(2,20)=-1.58(\mathrm{p} .13)$; Interés docente $\mathrm{t}(2,20)=$ -1.31 ( p .21); Interés alumno $\mathrm{t}(2,20)=-.17$ (p .86); Elevada aptitud matemática $\mathrm{t}(2,20)$ $=.81(\mathrm{p} .43)$; Alta capacidad detectada $\mathrm{t}(2,20)=.40(\mathrm{p} .69) ; \mathrm{BADyG}-\mathrm{E} 3 \mathrm{t}(2,20)=-.84(\mathrm{p}$ .41). Los resultados para los indicadores de aptitud no estarían afectados por el sexo de los participantes. En palabras de la UNESCO (2019, p.72):

La desventaja de las niñas no se basa en la capacidad cognitiva, sino en los procesos de socialización y de aprendizaje dentro de los cuales se las cría y que dan forma a su identidad, creencias, conductas y elecciones de vida. "Descifrar el código" para descubrir estos factores es fundamental para crear más sendas de aprendizaje para las niñas y las mujeres en las disciplinas STEM (Ciencias, Tecnología, Ingeniería y Matemáticas por sus siglas en inglés).

\section{Discusión y conclusiones}

La competencia matemática es fundamental en el currículum escolar. Su importancia radica en lo básico de sus contenidos para desarrollar aprendizajes nuevos, en su transversalidad por su interrelación con el resto de las áreas curriculares y en su carácter práctico y funcional. Todo ello justifica su trascendencia, su integración en los procesos de enseñanza y aprendizaje y la importancia de su diagnóstico y de su desarrollo.

En el curso 2011/2012 fueron diagnosticados mediante la BECOMA 22 estudiantes con nivel superior de competencia matemática. Predominaba el sexo masculino (64\%) y su escolarización principal era en colegios urbanos de titularidad pública. No hubo escolares repetidores de curso y el rendimiento general en Matemáticas era de sobresaliente. Fueron descritos por su profesorado y por ellos mismos mayoritariamente con nivel máximo de interés y motivación hacia las Matemáticas y los promedios en el 
BADYG-E3 fueron de 92 como media de centil y 141 de CI. De ellos, solo dos estaban diagnosticados con altas capacidades.

En el curso 2017/2018 dicho grupo ha sido nuevamente evaluado en las mismas características que en 2011/2012. La consistencia de los resultados entre las medidas repetidas es alta y las diferencias observadas en ellas entre ambos periodos no alcanzan la significación estadística. Es decir, seis años después el grupo mantiene el alto rendimiento en Matemáticas y vuelve a ser descrito nuevamente por su profesorado y por ellos mismos con alto interés y motivación hacia las Matemáticas y con un buen promedio en el BADYG-S (centil promedio 93.32, CI medio 133.68). Estos resultados cabe interpretarlos como un aval a la validez diagnóstica de la BECOMA. De los 22 estudiantes, tres estaban diagnosticados con altas capacidades lo que vendría a significar una praxis restrictiva y un tanto rígida de la evaluación psicopedagógica que llevan a cabo.

Aunque las diferencias encontradas entre ambos periodos no alcanzan la significación estadística, los datos originales indican que, respecto a 2011/2012, en 2017/2018 los cambios producidos apuntan a un ligero descenso de los resultados de los estudiantes en las características consideradas, tanto en la prueba psicopedagógica BADyG (BADyG-E3 y BADyG-S) como en el rendimiento académico en Matemáticas y en el interés y motivación hacia el área de Matemáticas según el punto de vista del docente y del propio escolar. El leve descenso afecta más a las chicas que a los chicos. Así, el rendimiento en Matemáticas ha descendido en la mitad de la muestra femenina y también parecen afectarle más los leves descensos motivacionales y de confianza en sí mismas, si bien el sexo no arroja diferencias significativas entre los dos periodos en las características medidas. Sería interesante observar si ambos sexos optan a medio plazo por carreras del ámbito STEM.

Por otro lado, los juicios y estimaciones del profesorado sobre la capacidad matemática de los estudiantes y sobre su motivación e interés hacia esta área de conocimientos y hacia la alta capacidad de los estudiantes, fluctúan de un periodo a otro, lo que parece indicar dificultades en el profesorado para la identificación de las potencialidades del alumnado más capaz. No sería de extrañar dado que estos estudiantes vienen siendo invisibles en la escuela y, particularmente, las niñas. Un estudio reciente (Pérez \& Jiménez, 2018) señala que estos estudiantes no se mencionan explícitamente en el Plan de Atención a la Diversidad de los centros educativos pese a ser el instrumento básico para planificar, implementar y evaluar las medidas de atención a la diversidad; que un alto porcentaje de profesores dice que en sus centros no existen escolares con alta capacidad y que caso de existir, no sabrían reconocerlos. Por otra parte, la baja prevalencia de estudiantes diagnosticados con alta capacidad a nivel nacional, el 0,33\% en el curso 2016/2017 (Ministerio de Educación y Formación Profesional, 2019), nos lleva a proponer la revisión de estas prácticas en la escolaridad obligatoria a fin de que el punto de corte sea más amplio y la escuela pueda fomentar tempranamente la alta capacidad, particularmente, la de los estudiantes procedentes de los entornos sociales más precarios. Gagné (2015) considera que sobre el $10 \%$ sería un punto de corte razonable. Remover las malas prácticas equivale a reconocer que la alta capacidad no está reñida con repetir curso (práctica que debería ser sumamente excepcional), con tener descenso del rendimiento o rendimiento nor- 
mal o con evaluarse con excesiva autocrítica como suele ser el caso de la población femenina. Como todos los estudiantes están sometidos a los vaivenes de la vida y tienen que afrontar, además, algunos retos sociales y emocionales específicos debido a su capacidad (Neihart, Pfeiffer, \& Cross, 2015).

También cabe concluir sobre la importancia de la trayectoria escolar. Se ha constatado que los escolares con mayor rendimiento no siempre se muestran más motivados intrínsecamente por la materia. En el caso de la competencia matemática, profundizar en el dominio afectivo matemático debería ser un objetivo educacional por sí mismo (Palacios, Arias, \& Arias, 2014). El docente tendrá que atender a los intereses y motivaciones cara a prevenir y reducir el rechazo y el fracaso escolar que esta área genera (Choi \& Calero, 2013; Guven \& Cabakcor, 2013). Su formación competencial (Méndez, Méndez, \& Fernández-Río, 2015) y matemática (Cleary \& Chen, 2009; Gutiérrez-Gutiérrez, Gómez, \& Rico, 2016; Nortes \& Nortes, 2016; Rico, Gómez, \& Cañadas, 2014). será también importante en la consecución de estos fines. Y deberá de ser consciente de que la prioridad de los procesos educativos recae sobre el estudiante y su aprendizaje y no sobre el propio docente y su metodología de enseñanza.

El contenido de este artículo tiene relevancia escolar y social y parece obligado replantearse, por un lado, la importancia del diagnóstico y la delimitación de características distintivas de los escolares con altas capacidades y, por otro, la generalización de innovaciones y mejoras escolares y sociales que el trabajo con ellos puede acarrear a corto, medio y largo plazo. Los criterios e indicadores utilizados en esta investigación son tradicionalmente usados para detectar las potencialidades del alumnado y, por ende, criterios a tener presentes en estudios de índole pedagógica. La generalización de investigaciones de este tipo es imprescindibles ante la invisibilidad y el ostracismo de estos escolares en las aulas, problema más agudizado si cabe tras observar nuevamente, con un intervalo de seis años, las dificultades de los docentes para diagnosticar las capacidades superiores y dar apoyo desde la práctica escolar. Hay que hacer todo lo posible en educación por sacar a la luz sus potenciales.

Por último, otorgar aliento al profesorado por su participación en esta investigación y por su papel trascendente en los procesos educativos de los escolares más capaces. Su capacitación y su rol en el aumento del bienestar personal, académico y emocional de todo el alumnado, incluyendo los de rendimiento y capacidades superiores, es fundamental, más si cabe cuando hay desajustes entre lo que un estudiante hace o es capaz de hacer y lo que realmente ejecuta o transmite con su desempeño habitual.

\section{Referencias}

Almeida, L., \& Oliveira, E. (2010). Los alumnos con características de sobredotación: la situación actual en Portugal. Revista Electrónica Interuniversitaria de Formación del Profesorado, 13(1), 85-95.

Cerda, G., Ortega-Ruiz, R., Casas, J., Del Rey, R., \& Pérez, C. (2016). Predisposición desfavorable hacia el aprendizaje de las Matemáticas: una propuesta para su medición. Estudios Pedagógicos, 42(1), 53-63. Doi: 10.4067/s0718-07052016000100004 
Cerda, G., Romera, E.M., Casas, J.A., Pérez, C., \& Ortega-Ruiz, R. (2017). Influencia de variables cognitivas y motivacionales en el rendimiento académico en matemáticas en estudiantes chilenos. Educación XX1, 20(2), 365-385. Doi: 10.5944/educxx1.19052

Choi, A., \& Calero, J. (2013). Determinantes del riesgo de fracaso escolar en España en PISA 2009 y propuestas de reforma. Revista de Educación, 362, 562-593. Doi: 10.4438/1988-592X-RE-2013-362-242

Cleary, T.J., \& Chen, P.P. (2009). Self-regulation, motivation and math achievement in middle school: Variations across grade level and math context. Journal of School Psychology, 47(5), 291-314. Doi: 10.1016/j.jsp.2009.04.002

Cueli, M., García. T., \& González-Castro, P. (2013). Autorregulación y rendimiento académico en Matemáticas. Aula Abierta, 41(1), 39-48.

De La Rosa, J.M. (2007). Didáctica para la resolución de problemas. Andalucía: Junta de Andalucía.

Escudero, J.M., González, M.T., \& Rodríguez, M.J. (2013). La mejora equitativa de la educación y la formación del profesorado. Multidisciplinary Journal of Educational Research, 3, 206-234. Doi: 10.447/remie.2013.014

Escudero, J.M., González, M.T., \& Rodríguez, M.J. (2018). Los contenidos de la formación continuada del profesorado: ¿Qué docentes se están formando? Educación XX1, 21(1), 157-180. Doi: 10.5944/educxx1.20183

Gagné, F. (2015). De los genes al talento: la perspectiva DMGT/CMTD. Revista de Educación, 368, 12-39. Doi: 10.4438/1988-592X-RE-2015-368-289

García-Perales, R. (2014). Diseño y validación de un instrumento de evaluación de la competencia matemática. Rendimiento matemático de los alumnos más capaces. Tesis de Doctorado para la obtención del título de Doctor en Educación. Facultad de Educación. UNED. Recuperado de http://e-spacio.uned.es/fez/view/tesisuned:Educacion-Rgarcia

García-Perales, R., \& Jiménez, C. (2016) Diagnóstico de la competencia matemática de los alumnos más capaces. Revista de Investigación Educativa, 34(1), 205-219. Doi: 10.6018/rie.34.1.218521

García-Perales, R. (2017). Desempeño docente para la identificación de los más competentes para la matemática. Profesorado, Revista de currículum y formación del profesorado, 22(Extraordinario), 271-278.

Gasco, J. (2016). El empleo de estrategias en el aprendizaje de las matemáticas en Enseñanza Secundaria Obligatoria. Revista de Investigación Educativa, 34(2), 487-502. Doi: 10.6018/rie.34.2.222901

Geist, E. (2009). Children are born mathematicians: Supporting mathematical development, birth to age 8. Upper Saddle River, NJ: Pearson.

Gutiérrez-Gutiérrez, A., Gómez, P., \& Rico, L. (2016). Conocimiento matemático sobre números y operaciones de los estudiantes de Magisterio. Educación XX1, 19(1), 135 158. Doi: 10.5944/educxx1.15581

Guven, B., \& Cabakcor, B. (2013). Factors influencing mathematical problem-solving achievement of seventh grade Turkish students. Learning and Individual Differences, 23, 131-137. Doi: 10.1016/j.lindif.2012.10.003

Instituto Nacional de Evaluación Educativa (INEE) (2013). PISA 2012: Informe Español. Volumen I: Resultados y contexto. Madrid: Ministerio de Educación, Cultura y Deporte. 
Mato, M.D., Espiñeira, E., \& Chao, R. (2014). Dimensión afectiva hacia la matemática: resultados de un análisis en educación primaria. Revista de Investigación Educativa, 32(1), 57-72. Doi: 10.6018/rie.32.1.164921

Méndez, D., Méndez, A., \& Fernández-Río, F. (2015). Análisis y valoración del proceso de incorporación de las Competencias Básicas en Educación Primaria. Revista de Investigación Educativa, 33(1), 233-246. Doi: 10.6018/rie.33.1.183841

Ministerio de Educación y Formación Profesional (2019). Datos estadísticos no universitarios. Recuperado de http://www.educacionyfp.gob.es/servicios-al-ciudadano-mecd/ estadisticas/educacion/no-universitaria/alumnado/necesidades-apoyo/2016-17.html

Muñoz, J. M., \& Espiñeira, E. M. (2010). Plan de mejoras fruto de la evaluación de la calidad de la atención a la diversidad en un centro educativo. Revista de Investigación Educativa, 28(2), 245-266.

Muñoz, J.M., \& Mato, M.D. (2008). Análisis de las actitudes respecto a las Matemáticas en alumnos de ESO. Revista de Investigación Educativa, 26(1), 209-226.

Neihart, M., Pfeiffer, S., \& Cross, T. (2015). The Social and Emotional Development of Gifted Children. Prufrock Press: Service Publication of the National Association for Gifted Children. Segunda edición.

Nortes, A., \& Nortes, R. (2013). Formación inicial de maestros: un estudio en el dominio de las Matemáticas. Profesorado, Revista de currículum y formación del profesorado, 17(3), 185-200.

Nortes, R., \& Nortes, A. (2016). Resolución de problemas, errores y dificultades en el grado de maestro de primaria. Revista de Investigación Educativa, 34(1), 103-117. Doi: 10.6018/34.1.229501

Organización para la Cooperación y el Desarrollo Económico (OCDE) (2009). Top of the class. High performers in science in PISA 2006. París: PISA, OECD Publishing. Doi: 10.1787/9789264060777-en

OCDE (2013). PISA 2012 Assessment and Analytical Framework: Mathematics, Reading, Science, Problem Solving and Financial Literacy. París: PISA, OECD Publishing. Doi: 10.1787/9789264190511-en

OCDE (2016). PISA 2015: Resultados Clave. París: PISA, OECD Publishing.

Palacios, A., Arias, V., \& Arias, B. (2014). Attitudes Towards Mathematics: Construction and Validation of a Measurement Instrument. Revista de Psicodidáctica, 19(1), 67-91. Doi: 10.1387/RevPsicodidact.8961

Pérez, L., \& Jiménez, C. (2018). Influencia de la organización escolar en la educación de los estudiantes de altas capacidades. Enseñanza \& Teaching, 36(1), 151-178.

Renzulli, J. S., \& Gaesser, A. (2015). Un sistema multicriterial para la identificación del alumnado de alto rendimiento y de alta capacidad creativo-productiva. Revista de Educación, 368, 96-131. Doi: 10.4438/1988-592X-RE-2015-368-290

Rico, L., Gómez, P., \& Cañadas, M. (2014). Formación inicial en educación matemática de los maestros de Primaria en España, 1991-2010. Revista de Educación, 363, 35-59. Doi: 10.4438/1988-592X-RE-2011-362-152

Sastre, S. (2014). Intervención psicoeducativa en la alta capacidad: funcionamiento intelectual y enriquecimiento extracurricular. Revista de Neurología, 58, 89-98. Doi: 10.33588/rn.58s01.2014030 
Torres, J.A., \& Castillo, S. (2016). Incidencia de las políticas de apoyo educativo en las estructuras organizativas de los centros desde la perspectiva del profesorado: Un estudio en la provincia de Jaén. Educación XX1, 19(2), 205-228. Doi: 10.5944/ educxx1.16462

Tourón, J., Lizasoaín, L., Castro, M., \& Navarro, E. (2012). Alumnos de alto, medio y bajo rendimiento en Matemáticas en TIMSS. Estudio del impacto de algunos factores de contexto. PIRLS-TIMSS 2011: Informe Español. Análisis secundario, 2, 187-215.

UNESCO (2019). Descifrar el código: La educación de las niñas en ciencias, tecnología, ingeniería y matemáticas (STEM). Francia: UNESCO.

Zeichner, K., Payne, K., \& Brayko, K. (2015). Democratizing Teacher Education. Journal of Teacher Education, 66(2), 122-135. Doi: 10.1177/0022487114560908

Fecha de recepción: 7 de marzo de 2019.

Fecha de revisión: 26 de marzo de 2019.

Fecha de aceptación: 16 de diciembre de 2019. 